\title{
Impact of QW coupling on the binding energy in InGaN/GaN under the effects of the size, the impurity and the internal composition
}

\author{
Walid Belaid ${ }^{1,2}$, Haddou El Ghazi ${ }^{1,3, *}$, Izeddine Zorkani $^{1}$, and Anouar Jorio ${ }^{1}$ \\ ${ }^{1}$ FSDM, Mohammed Ben Abdellah University, Fes, Morocco \\ ${ }^{2}$ Laser Spectroscopy Group, Department of Physics, FS, University of Selcuk, 42031 Campus, Selcuklu, Konya, Turkey \\ ${ }^{3}$ ENSAM Laboratory, ENSAM, Hassan II University, Casablanca, Morocco
}

\begin{abstract}
In the present paper, the binding energy of hydrogenic shallow-donor impurity in simple and double coupled quantum wells based on unstrained wurtzite ( $\mathrm{In}, \mathrm{Ga}) \mathrm{N} / \mathrm{GaN}$ is investigated. Considering the effective-mass and dielectric mismatches between the well and its surrounding matrix, the numerical calculations are performed within the framework of the parabolic band and the single band effective-mass approximations under the finite potential barrier using finite element method (FEM). According to our results, it appears that the main effect of the wells coupling is to enhance the binding energy. It is also obtained that the binding energy is strongly sensitive to the internal and external parameters and can be adjusted by the quantum well/barrier width, the impurity position and the internal Indium composition. Our results are in good agreement with the finding especially for those obtained by the variational approach.
\end{abstract}

\section{Introduction}

Low-dimensional systems (LDS) based on single and multiple quantum well (SQW, MQW) quantum well wire (QWW) and quantum dot (QD) form a great domain for research both theoretically and experimentally [1-16]. During the few last decades, IIInitrides semiconductor systems such AlN, GaN, $(\mathrm{In}, \mathrm{Ga}) \mathrm{N}$ and $(\mathrm{Al}, \mathrm{Ga}) \mathrm{N}$ have emerged as the adequate materials for applications such solar cell, laser, diode laser. They present attractive properties like chemical and thermal stabilities, great radiation resistance and high absorption and also because the recent progress in quality of materials grown by different technique such beam epitaxy molecular (BEM) and metal organic chemical vapor deposition (MOCVD) [17-26].

It is well known that the impurity in such reduced system has a great impact on the electronic and optical properties. It is widely studied through several papers [27-32] theoretically and experimentally. For instance, based on photoluminescence and transmission electron microscopy, Mutta et al. have examined some internal and external parameters on the (In,Ga)N/GaN SQW electronic properties [27]. The internal composition effects on the optical properties have reported for wurtzite (In, Ga)N/GaN QW [28]. The finite barrier and built-in electric field influences are investigated on the SQW [8]. In the same trend, the energy difference between the implied states in SQW under BEF is reported by Chi et al [33]. It is demonstrates that the energy transition is strongly-dependent on the structure size, composition, BEF and the impurity. But, to the best of our knowledge, (In,Ga)N/GaN coupled QW remains less commonly used and studied as active systems. To the best of our knowledge, no work has done to treat the coupling effect on the electronic properties based on the finite element method (FEM).

In this paper, the effects of impurity's position, structure size, In-composition and coupling effects are numerically investigated on the ground-state binding energy based on the FEM.

\section{Theoretical framework}

Let us to consider a hydrogenic shallow-donor impurity situated at $x_{0}=L+\frac{l}{2}$. The indium fraction in the composite material is denoted $v$ while $l$ is the width of the each well and $L$ is the width of the outer barrier and $L_{1}$ is the width of the coupling barrier of SQW (DQW) wurtzitestructure with $\mathrm{GaN} / \mathrm{In}_{v} \mathrm{Ga}_{1-v} \mathrm{~N}$ composition. The schematic description of the structures under study are given in Fig. 1. In this paper, the results are given in the effective units, i.e., the effective Rydberg energy $\mathrm{R}^{*}=\frac{\mathrm{m}^{*} \mathrm{e}^{4}}{2 \hbar^{2} \varepsilon_{0}^{2}}$ as the unit of the energyand the effective Bohr radius $\mathrm{a}^{*}=\frac{\varepsilon_{0} \hbar^{2}}{\mathrm{~m}^{*} \mathrm{e}^{2}}$ as the unit of length. Within the framework of single-band effective-mass and the parabolic band approximations, the Hamiltonian of the electron in the presence of a shallow-donor impurity in structures mentioned in Fig.1 can be written as:

\footnotetext{
*Corresponding author: hadghazi@gmail.com
} 

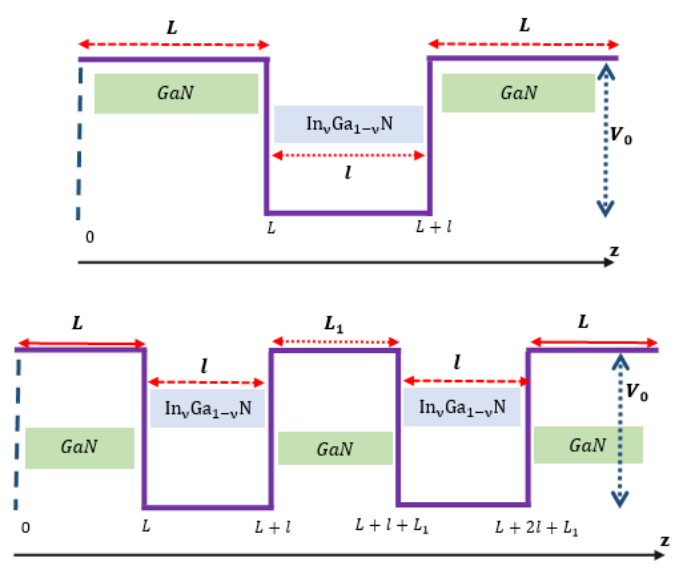

Fig. 1.Single $\mathrm{GaN} / \mathrm{In}_{v} \mathrm{Ga}_{.2} \mathrm{~N}$ QW and double coupled GaN $/ \mathrm{In}_{.2} \mathrm{Ga}_{.8} \mathrm{~N} / \mathrm{GaN} / \mathrm{In}_{.2} \mathrm{Ga}_{.8} \mathrm{~N} / \mathrm{GaN}$ QWs description under study.

$$
\mathrm{H}=-\frac{\hbar^{2}}{2 \mathrm{~m}^{*}} \nabla^{2}-\frac{e^{2}}{\varepsilon^{*}\left|\overrightarrow{\mathrm{r}}-\overrightarrow{\mathrm{r}}_{0}\right|}+\mathrm{V}(\mathrm{z})
$$

$e$ is the electron charge, $\quad\left|\overrightarrow{\mathrm{r}}-\overrightarrow{\mathrm{r}}_{0}\right|=$ $\sqrt{\left(x-x_{0}\right)^{2}+\left(y-y_{0}\right)^{2}+\left(z-z_{0}\right)^{2}}$ is the electronimpurity distance and $\mathrm{V}(\mathrm{z})$ is the finite potential barrier given as:

$$
\mathrm{V}(\mathrm{z})=\left\{\begin{array}{cr}
0 & \text { well } \\
V_{0} & \text { barrier }
\end{array}\right.
$$

$\mathrm{V}_{0}=\mathrm{Q} . \Delta \mathrm{E}_{\mathrm{g}}(\mathrm{x})$ denotes the potential barrier, whereas $\mathrm{Q}(=0.7)$ and $\Delta \mathrm{E}_{\mathrm{g}}$ are respectively the conduction band off-set parameter nd the difference between the band gaps of GaN and $\operatorname{In}_{v} \mathrm{Ga}_{1-v} \mathrm{~N}$ which are defined as follows:

$$
\Delta E_{g}(v)=E_{g}(G a N)-E_{g}\left(\operatorname{In}_{v} \mathrm{Ga}_{1-v} \mathrm{~N}\right)
$$

For $I n_{v} G a_{1-v} N$, the band-gap energy is obtained as the interpolation between InN and GaNcorrected by the bowing parameter [34]:

$$
\begin{gathered}
E_{g}\left(I n_{v} G a_{1-v} N\right)= \\
v \cdot E_{g}(\operatorname{InN})+(1-v) \cdot E_{g}(G a N)-b \cdot(1-v) \cdot v
\end{gathered}
$$

$\varepsilon^{*}$ and $m^{*}$ are respectively the main relative dielectric constant and the effective mass. For $(\operatorname{In}, G a) N$, they are equal to the linear combination of those of InN and GaN.

$$
\begin{gathered}
m^{*}= \\
\begin{array}{c}
v \cdot m_{I n N}^{*}+(1-v) \cdot m_{G a N}^{*}-b \cdot(1-v) \cdot v \quad \text { well } \\
m_{G a N}^{*}
\end{array} \\
\varepsilon^{*}=\left\{\begin{array}{cc}
v \cdot \varepsilon_{I n N}^{*}+(1-v) \cdot \varepsilon_{G a N}^{*}-b \cdot(1-v) \cdot v \\
\varepsilon_{G a N}^{*}
\end{array}\right. \text { well }
\end{gathered}
$$

The electron effective-mass in $\mathrm{GaN}(\mathrm{InN})$ is $m_{\text {GaN }}^{*}=$ $0.19 m_{0}\left(m_{I n N}^{*}=0.1 m_{0}\right) m_{0}$ is the free electron mass. The mean dielectric constant is $\varepsilon_{G a N}^{*}=9.8 \varepsilon_{0}\left(\varepsilon_{\text {InN }}^{*}=\right.$ $\left.13.7 \varepsilon_{0}\right)$ for $\mathrm{GaN}(\mathrm{InN})$ where $\varepsilon_{0}$ is the static electrical permittivity of the vacuum.
For the dielectric constant at the interfaces, we have adopted the expression:

$$
\varepsilon(\text { Interfaces })=\sqrt{\varepsilon(G a N) * \varepsilon\left(I n_{v} G a_{1-v} N\right)}
$$

Using the effective units, the Hamiltonian described in Eq. (1) becomes:

$$
H=-\nabla^{2}-\frac{2}{r}+V(z)
$$

The eigenvalues of the Hamiltonian given in Eq. (8) can be obtained with a numerical method using the finiteelement method. The ground-state binding energy is obtained as follows:

$$
E_{b}=E_{0}-E_{I}
$$

Where $E_{I}\left(E_{0}\right)$ is the ground state energy in the presence (absence) of the impurity.

\section{Results and discussion}

The binding energy of hydrogenic shallow-donor impurity in simple and double quantum wells based on unstrained wurtziteGaN/(In, Ga)N/GaN is calculated as a function of the quantum well/barrier width, the impurity position and the well coupling. Our calculations are performed based on the finite element method.

To investigate the influence of the well and barrier widths and the impurity position, we have primarily presented in Fig. 2 the binding energy of a wurtzite (In,Ga)N/GaN SQW versus the well width. For all impurity's position and barrier's width, the binding energy increases with decreasing the well width until it reaches a maximum corresponding to the critical value $\left(l_{c}\right)$ of the well size around the effective bohr radius. This is due to diminishing of the electron-impurity distance which enhances the binding energy. For the strong confinement regime $\left(l<l_{c}\right)$, the majority of the wave-function is in the barrier region. Therefore, the electron is less attracted by the impurity which is manifested by the reduction of the binding energy tending to the GaN bulk value. According to the same figure, the binding energy shows two behaviors limited by the width critical value. The binding energy drops (augments) with increasing the barrier width for strong (low) confinement regime. The physical reason is that for strong confinement, the whole of the wave-function is in the barrier and then the barrier width increasing induces its more spreading in the barrier which reduces the binding energy. For low confinement, with increasing the barrier width the wave-function spread in the barrier diminishes and then the binding energy can only enhanced.

The inset figure displays the results concerning the effects of the impurity's position on the binding energy for a fixed value of the barrier width. With the displacement of the impurity far away the structure center the electron-impurity distance increases and therefore the binding energy shows a significant drop. It is clearly seen that this effect is more marked especially 
in the moderate regime confinement than in the strong one.

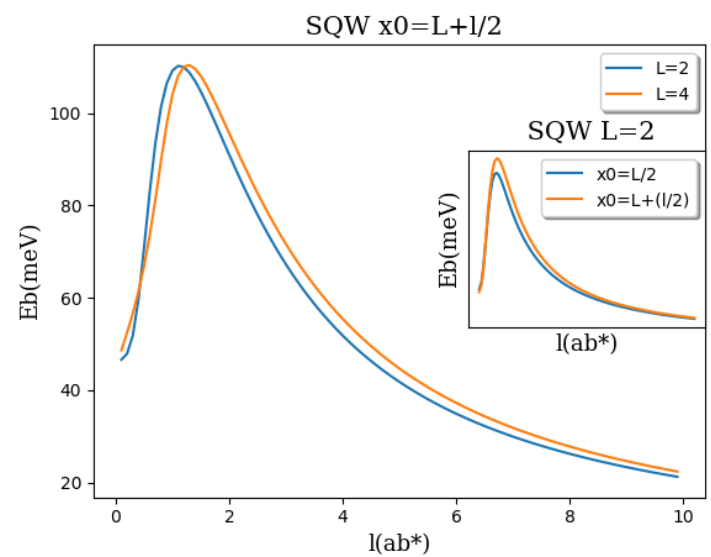

Fig. 2. Binding energy in $\mathrm{GaN} /(\mathrm{In}, \mathrm{Ga}) \mathrm{N} / \mathrm{GaN} \mathrm{SQW}$ as a function of the well width. The effects of the barrier width and the impurity's position are included.

Binding energy in DQW for two different barrier widths and impurity's position are displayed in figure 3 for a fixed coupling width $L_{1}=2$. It is reaveled that the behavior of the binding energy in DQW versus the well size is the same as that of the SQW. The principle characteristic is that the binding energy in DQW is more greater than that in SQW. This enhancement can be assigned to the coupling effect. For coupling barrier size equals to $L_{1}=2$, the improvement rate is about $77 \%$. In the same trend, the impact of the impurity's position is more significant in DQW in particularly for the moderate confinement regime. The same figure (inset) contains the variation of the binding energy versus the well width for two different values of the impurity position. It is clearly seen that as the impurity moves from the left barrier to structure center, the binding energy is strongly enhanced. For instance, the rate enhancement is about $36 \%$ when it is moved from $L$ to $L+l / 2$.

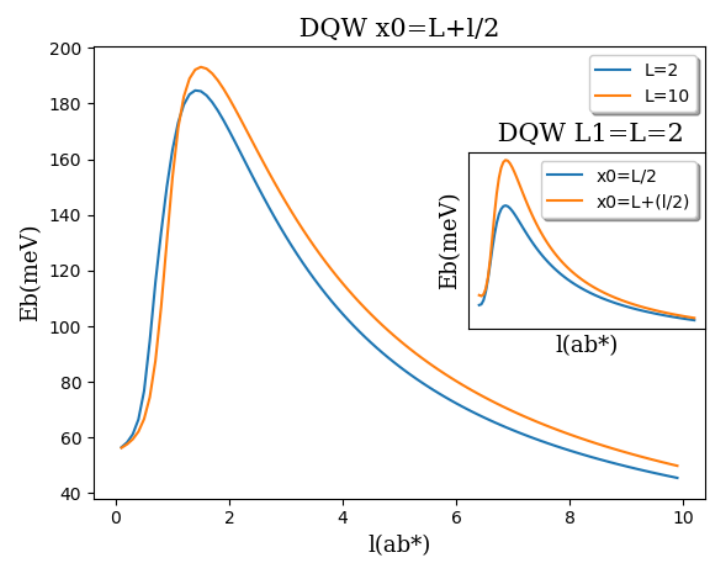

Fig. 3.Binding energy in

$\mathrm{GaN} /(\mathrm{In}, \mathrm{Ga}) \mathrm{N} / \mathrm{GaN} /(\mathrm{In}, \mathrm{Ga}) \mathrm{N} / \mathrm{GaN} \mathrm{DQW}$ as a function of the well width. The effects of the barrier width and the impurity's position are included.

Figure 4 depicts the results corresponding to groundstate the binding energy in double QWs as a function of the well width under the effects of the In-composition in the well. For instance, we have reported the results for $x=0.1,0.2$ and 0.3 for $L_{1}=L=2$. As expected, two behaviors are obtained. On the one hand, for low confinement regime, the binding energy dropps according to the internal composition. The main reason of this change can be explained by the diminishing of the coupling effect, i.e., the increasing of Indium fraction in the wells leads to the enhancement of the potential barrier which induces more confinement of the electron wave-function and then the binding energy can only go down. On the other hand, for the strong confinement regime it is found that the binding energy is enhanced versus In-composition in the wells. As known, in such regime the wave-function is more spread in barriers, so, the In-fraction increasing improves this confinement which enhances the binding energy.

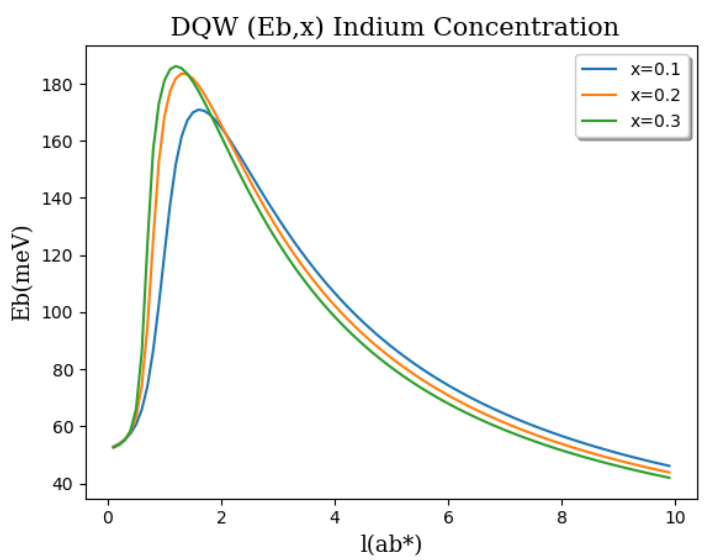

Fig. 4. Ground-state binding energy according to well width for three different values of the Indium concentrations.

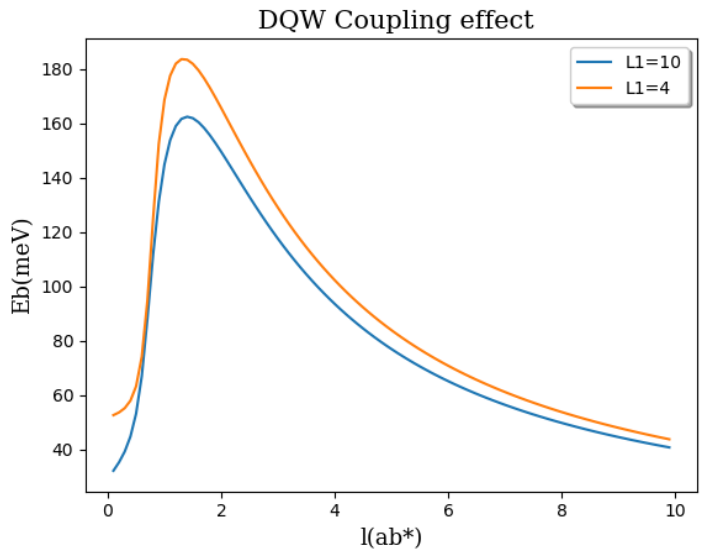

Fig. 5. Ground-state binding energy in DQW as a function of the well width for two different values of the coupling barrier width.

Finally, the variation of the ground-state binding energy in DQW according to well width under the effect of the coupling barrier width is depicted on figure 5 . The calculation includes $x=0.2, L=10$ and $x_{0}=L+$ $l / 2$ as fixed parameters and two different values of the 
intermidiatry barrier. It is observed that as the coupling barrier width increases the binding energy drops. We notice that the rate drop is about $20 \mathrm{meV}$ representing $12.5 \%$ of the binding energy. This result can be assigned to the reduction of the wavefunctionsoverlapping in both wells.

\section{Conclusion}

In this work, based on the finite element method we have investigated the impurity's position, the Indium composition, the structure size and the coupling effects on the ground-state binding energy in SQW and DQW. The wave-function and the energy level are calculated within the framework of the effective mass approximation. The overall conclusion of this paper is that the binding energy is strongly depending on these parameters. Depending on the particular nature of the varying internal parameters, we found that:

- The binding energy is enhanced versus the coupling effect.

- It is reduced as a function of displacement of the impurity far away the structure center.

- The In-fraction impact depends on the confinement regime.

\section{References}

1. B. Gill, Group III-nitride Semiconductor Compounds, Clarendon Press, Oxford, (1998).

2. S. Nakamura, S.F. Chichibu, Introduction to Nitride Semiconductor Blue Lasers and Light Emitting Diodes, Taylor and Francis, London, (2000).

3. N. Esuanu, E.C. Niculescu, UPB Sci. Bull. 72, 21 (2010)

4. C.A. Duque, E. Kasapoglu, S. Sakiroglu, H. Sari, E. Sokmen, Appl. Surf. Sci. 256, 740 (2010)

5. P. Baser, S. Elagoz, N. Baraz, Physica E 44, 356 (2011)

6. H. El Ghazi, A. Jorio, I. Zorkani, Phys. B: Condens. Matter 410, 49 (2013)

7. H. El Ghazi, A. John Peter, Phys. B 470-471, 64-68 (2015)

8. M. S, ahin, Phys. Rev. B 77, 045317 (2008)

9. A. Sali, H. Satori, M. Fliyou, H. Loumrhari, Phys. Status Solidi (b) 232, 209 (2002)

10. W. Xie, SuperlatticesMicrostruct. 65, 271 (2014)

11. H. El Ghazi, A. John Peter, Solid State Comm. 201, 5 (2015)

12. M. Jin, W. Xie, T. Chen, Superlattices. Microstruct. 62, 59 (2013).

13. W. Xie, Superlattices. Microstruct. 58, 94 (2013)

14. W. Xie, Phys. Lett. A 377, 903 (2013)

15. H. El Ghazi, A. Jorio, I. Zorkani, Superlattices Microstruct. 71, 211-216 (2014)
16. E.C. Niculescu, M. Cristea, J. Lumin. 135, 120 (2013)

17. L.M. Burileanu, J. Lumin. 145 (2014) 684.

18. M.G. Barseghyan, A.A. Kirakosyan, C.A. Duque, Eur. Phys. J. B 72, 521 (2009)

19. M.G. Barseghyan, M.E. Mora-Ramos, C.A. Duque, Eur. Phys. J. B 84, 265 (2011)

20. S. Baskoutas, A.F. Terzis, J. Comput. Theor. Nanosci.7, 492 (2010)

21. C.A. Duque, E. Kasapoglu, S. Sakiroglu, H. Sari, E. Sokmen, Appl. Surf. Sci. 257 (2011) 2313.

22. P.G. Moses, C.G. Van De Wall, Appl. Phys. Lett. 96, 021908 (2010)

23. P. Baser, S. Elagoz, D. Kartal, H.D. Karki, Superlatt. Microstru. 49(2011) 497.

24. P. Baser, I. Altuntas, S. Elagoz, Fen BilimleriDerg. 23, 171 (2011)

25. H. El Ghazi, I. Zorkani, A. Jorio, Phys. B: Condens. Matter 412, 87 (2013)

26. H. El Ghazi, A. Jorio, I. Zorkani, Phys. B: Condens. Matter 426, 155 (2013)

27. G.R. Mutta, P. Ruterana, J.L. Doualan, M.P. Chauvat, F. Ivaldi, S. Kret, N.A.K. Kaufmann, A. Dussaigne, D. Martin, N. Grandjean, Phys. Status Solidi B 248, 1187 (2011)

28. D.M. Graham, A. Soltani-Vala, P. Dawson, M.J. Godfrey, T.M. Smeeton, J.S. Barnard, M.J. Kappers, C.J. Humphreys, E.J. Thrush, J. Appl. Phys. 97, 103508 (2005)

29. H. Masui, H. Yamada, K. Iso, S. Nakamura, S.P. DenBaars, J. Phys. D: Appl. Phys. 41, 225104 (2008)

30. H. Yamada, K. Iso, M. Saito, H. Masui, K. Fujito, S.P. DenBaars, S. Nakamura, Appl. Phys. Express 1041101 (2008)

31. C. Xia, Y. Jia, S. Wei, H.N. Spector, J. Lumin. 132, 607 (2012)

32. L. Guo, X. Wang, H. Xiao, B. Wang, J. Cryst. Growth 298, 522 (2007)

33. P. Bigenwald, P. Lefebvre, T. Bretagnon, B. Gill, Phys. Status Solidi B 216, 371 (1999)

34. H. El Ghazi, A. Jhon Peter, Superlattices and Microstructures 104, 222 (2017) 